\title{
Prática de atividades físicas e custo do tratamento ambulatorial de diabéticos tipo 2 atendidos em unidade básica de saúde
}

\author{
Physical activity and healthcare cost of type 2 diabetic \\ patients seen at basic units of healthcare
}

Jamile Sanches Codogno', Rômulo Araújo

Fernandes ${ }^{1,2}$, Henrique Luiz Monteiro ${ }^{1,3}$

\section{RESUMO}

'Programa de Pós-Graduação em Ciências da Motricidade, Instituto de Biociências, Universidade Estadual Paulista (Unesp), Rio Claro, SP, Brasil 2 Departamento de Educação Física, Unesp, Presidente Prudente, SP, Brasil

${ }^{3}$ Departamento de Educação

Física, Unesp, Bauru, SP, Brasil

Correspondência para: Rômulo Araújo Fernandes Departamento de Educação Física, Universidade Estadual Paulista Rua Roberto Simonsen, 305 19060-900 - Presidente Prudente, SP, Brasil romulo_ef@yahoo.com.br

Recebido em 7/Fev/2011 Aceito em 5/Jan/2012
Objetivos: Observar se existem diferenças nos valores monetários destinados aos procedimentos de saúde para o tratamento de pacientes diabéticos tipo 2 quando estratificados em diferentes níveis de atividade física habitual. Sujeitos e métodos: Cento e vinte um diabéticos tipo 2 foram avaliados em duas unidades básicas de saúde de Bauru, SP. Atividade física foi avaliada por meio de entrevista. Retroagindo um ano ao dia da avaliação, por meio de notas fiscais, foram computados valores de exames, medicamentos e consultas médicas e de enfermagem. Resultados: Quando comparados aos diabéticos ativos, os sedentários apresentaram gastos com consultas em clínico-geral $63 \%$ superiores $(p=0,017)$. Gastos com medicamentos para 0 tratamento de outras doenças também foram superiores em diabéticos sedentários $(p=0,001)$. Conclusões: Quando comparados de acordo com a prática de atividades físicas, diabéticos tipo 2 com menor prática de atividades físicas apresentam maiores custos com serviços médicos e consumo de medicamentos. Arq Bras Endocrinol Metab. 2012;56(1):6-11

Descritores

Diabetes mellitus tipo 2; atividade motora; análise de custo; Brasil

\section{ABSTRACT}

Objectives: To observe differences in healthcare costs according to the level of physical activity in type 2 diabetes mellitus. Subjects and methods: A total of 121 type 2 diabetic patients were interviewed in two basic units of healthcare in Bauru, Brazil. Physical activity level was assessed by a questionnaire carried out during an interview. Costs of exams, medications and doctor and nurse visits were assessed for the previous year using invoices obtained from basic units of healthcare. Results: Sedentary type 2 diabetic patients presented higher costs for doctor visits $(63 \% ; p=0.017)$ and medication to treat diseases other than diabetes $(p=0.001)$ than active patients. Conclusions: Sedentary type 2 diabetic patients presented higher healthcare costs related to doctor visits and medicine purchase. Arq Bras Endocrinol Metab. 2012;56(1):6-11

\section{Keywords}

Type 2 diabetes mellitus; motor activity; costs analyzis; Brazil

\section{INTRODUÇÃO}

$\mathrm{E}$ stima-se que, em todo o mundo, até 2025, 333 milhões de pessoas ( $8 \%$ da população adulta) apresentarão diagnóstico positivo para diabetes melito (DM) (1). Confirmando essas previsões, nos últimos 40 anos, os custos destinados ao tratamento do DM têm aumen- tado de forma alarmante em diferentes grupos populacionais $(2,3)$.

Nesse sentido, a utilização de terapia medicamentosa representa custo elevado e pode gerar efeitos colaterais, situação que se agrava quando observada a conduta terapêutica adotada pela rede pública de saúde (mantida 
pelos municípios com recursos do Sistema Único de Saúde - SUS) que não utiliza fármacos de última geração em razão do elevado custo do tratamento. Outro aspecto alarmante é o fato de que pacientes que fazem uso de medicamentos, muitas vezes, o utilizam em quantidades inadequadas, situação que é confirmada por Assunção e cols. (4) ao observarem que 41\% dos usuários de hipoglicemiantes orais não utilizavam a dose recomendada.

Por ser o tratamento medicamentoso de alto custo e apresentar contraindicações e problemas no entendimento quanto à dosagem adequada, a Sociedade Brasileira de Diabetes (5) apresenta estratégias de intervenções não medicamentosas que devem ser adotadas em primeira instância, sendo o tratamento medicamentoso iniciado quando mudanças no estilo de vida (controle alimentar e práticas de atividades físicas) não são suficientes para o controle da doença.

Nesse contexto, a prática de exercícios físicos, sobretudo os aeróbios, exerce influência significativa no combate ao DM tipo 2 (DM2). Sua relação com a doença ocorre devido à ação direta sobre indicadores, como colesterol total e lipoproteína de baixa densidade (LDL), na qual indivíduos com escores mais elevados de atividade física apresentam menores concentrações sanguíneas dessas estruturas (6). Esse poder depressor da atividade física deve-se à maior utilização dos lipídeos circulantes como fonte de energia na realização das tarefas motoras, bem como após o término do trabalho muscular, com o intuito de restabelecer os estoques energéticos. Além disso, durante a ação dos mecanismos acima citados, existe subsequente aumento de sensibilidade da membrana celular à ação insulínica, fator que diminui as concentrações de insulina e glicose circulantes (7).

Essas alterações fisiológicas caracterizam a atividade física como meio não farmacológico importante no combate ao DM2, entretanto, existem poucas evidências na literatura que apontem se os benefícios fisiológicos do exercício são suficientes para reduzir a demanda dos procedimentos de saúde e respectivos custos das condutas ambulatoriais. A primeira pesquisa nacional a analisar a relação da prática de atividades físicas e custos relacionados à saúde foi realizada somente em 2006 (8) e evidenciou menor consumo de medicamentos entre pessoas ativas. Por outro lado, não existe informação similar em populações diabéticas.

Dessa maneira, o objetivo do presente estudo foi observar se existem diferenças nos valores monetários destinados aos procedimentos de saúde para o tratamento de pacientes diabéticos tipo 2, atendidos por Unidade Básica de Saúde (UBS), quando estratificados em diferentes níveis de atividade física habitual.

\section{SUJEITOS E MÉTODOS}

Trata-se de estudo de corte transversal com características retrospectivas, realizado em Bauru, SP. As avaliações/entrevistas foram efetuadas em duas UBS: Vila Cardia e Otávio Rasi (ambas administradas pela Prefeitura Municipal), e foram avaliados 121 pacientes. Foi solicitada permissão prévia da secretaria de saúde para ter acesso aos prontuários clínicos e, também, solicitado local adequado para a instalação da equipe de avaliação, no caso, uma sala de consulta médica. Quanto aos usuários do serviço de saúde, as avaliações ocorreram após triagem realizada pelas enfermeiras, que analisavam o prontuário do paciente e sua disponibilidade em participar da pesquisa. O protocolo de investigação foi aprovado pelo Comitê de Ética e Pesquisa da Universidade Estadual Paulista, Campus de Rio Claro, Protocolo n. ${ }^{\circ}$ 6898-2008, bem como pelo Conselho de Ética da Secretaria Municipal de Saúde.

Para determinar os custos com o tratamento do DM2, a despesa total da UBS com cada paciente foi averiguada pela demanda por serviços que foram registrados nos prontuários. Retroagindo um ano à data da avaliação, buscou-se obter as seguintes informações: medicamentos fornecidos pelo serviço de saúde, exames laboratoriais realizados e o número de consultas. Para transformar os procedimentos em moeda corrente, utilizou-se a tabela do SUS para os valores dos exames laboratoriais e de médico especialista. Notas fiscais disponibilizadas pelas unidades básicas de saúde foram utilizadas para computar os valores unitários dos medicamentos fornecidos aos pacientes. Estudo prévio realizado na cidade em questão foi usado como referência para valores de consulta médica (clínico-geral), de enfermagem e de nutricionista, sendo o custo desses procedimentos fixados em R\$ 7,55 por atendimento (9). Todos os valores monetários apresentados no presente estudo foram calculados com uma base populacional de 100 diabéticos (valores multiplicados por 100).

As variáveis sexo, idade, tabagismo, uso regular de insulina e excesso de peso (valores de peso e estatura foram aferidos e o índice de massa corporal [IMC] foi calculado) foram utilizadas para indicar o perfil da população. Esses dados foram obtidos por meio de en- 
trevista estruturada, na qual o coordenador da pesquisa preencheu formulário com os dados dos pacientes.

A prática habitual de atividades físicas foi determinada pelo questionário proposto por Baecke e cols. (10), o qual foi preenchido pelo coordenador da pesquisa por meio de entrevista individual. O protocolo é composto por 16 questões fechadas que representam a prática de atividades distribuída em três domínios de atividades físicas: i) ocupacionais; ii) esportivas; e, iii) lazer. Por meio da aplicação do referido instrumento, foi possível determinar o escore de cada domínio da atividade física, e a soma dos escores de cada seção compõe um valor total adimensional, ou seja, a atividade física habitual. Para classificação da atividade física habitual, utilizou-se fórmula proposta por Baecke e cols. (10). Os pacientes avaliados foram subdivididos em quartil de acordo com o escore total individual fornecido pelo instrumento, os quais resultaram nos seguintes grupos de atividades físicas: Sedentários ( $1^{\circ}$ quartil); Moderadamente Ati$\operatorname{vos}\left(\mathrm{M}\right.$. Ativos $\left[2^{\circ}\right.$ e $3^{\circ}$ quartis]); e Ativos ( $4^{\circ}$ quartil).

Anteriormente ao início das análises estatísticas, para identificar possíveis fatores de confusão, considerou-se a relação de todos os diferentes indicadores de custo envolvidos no estudo com as variáveis (i) idade, (ii) tabagismo e (iii) IMC. Os resultados indicaram que não houve relação significativa entre as variáveis envolvidas, sem necessidade, portanto, de ajustar as subsequentes análises. Desse modo, para variáveis numéricas, o teste $t$ de Student para amostras independentes e a análise de variância (ANOVA) one-way (post-hoc de Tukey) foram empregados. Os cálculos foram realizados por meio do software Statistical Package for the Social Sciences (SPSS - versão 13.0), e um nível de significância de $5 \%$ foi utilizado em todos os procedimentos estatísticos realizados no estudo.

\section{RESULTADOS}

Foram analisados os dados de 121 diabéticos do tipo 2, todos pacientes de duas UBS localizadas na cidade de Bauru, SP. As primeiras análises realizadas tiveram o objetivo de verificar possíveis diferenças entre as UBS dos bairros "Otávio Rasi" e "Vila Cárdia". Não foram identificadas diferenças significativas para as variáveis sexo, grupo étnico e uso de tabaco. Foi encontrada diferença apenas para idade (UBS-Otávio Rasi 58,6 anos versus UBS-Vila Cardia 62,2 anos; teste $t$ com $\mathrm{p}=0,028$ ), porém, essa variável não se relacionou com os custos totais analisados. Essa similaridade entre os diabéticos de ambas as UBS permitiu conduzir o tratamento es- tatístico considerando a casuística das duas UBS como um único grupo de pacientes.

Da casuísta analisada, 40,5\% eram do sexo masculino $(\mathrm{n}=49)$ e $59,5 \%$, do feminino $(\mathrm{n}=72)$. Na amostra total, 11,6\% dos diabéticos declararam ser fumantes e $36,4 \%$ faziam uso regular de insulina. Uso de insulina $(\mathrm{p}=0,722)$ e tabagismo $(\mathrm{p}=0,662)$ não foi associado com os grupos de atividade física. A média total de idade da casuística foi de $60 \pm 9$ anos (limite inferior $-32,7$ anos; limite superior 74,9 anos) e não diferiu entre os grupos de atividade física (Sedentários: 61,2 \pm 11 anos; M. ativos: 59,8 7 anos; Ativos: 59,3 \pm 8 anos com $\mathrm{p}=$ $0,688)$. Padrão similar foi observado para os valores de IMC (Sedentários: $31,8 \pm 8 \mathrm{~kg} / \mathrm{m}^{2} ;$ M. ativos: $30,2 \pm 6$ $\mathrm{kg} / \mathrm{m}^{2}$; Ativos: $\left.30,3 \pm 5 \mathrm{~kg} / \mathrm{m}^{2} \operatorname{com} \mathrm{p}=0,507\right)$.

A figura 1 apresenta os valores de procedimentos de atenção básica a cada 100 pacientes, segundo os respectivos níveis de atividade física. Adotando-se o custo do grupo Ativo como referência, estabeleceu-se comparação entre os diferentes indicadores de custo. De maneira geral, embora em quase todos os procedimentos o grupo sedentário tenha apresentado valores superiores ao grupo ativo, observou-se que somente o custo com clínico-geral foi significativo na comparação entre os grupos Ativos e Sedentários (63\% superiores para os sedentários [ANOVA com $\mathrm{p}=0,017$ e o teste post hoc de Tukey com $\mathrm{p}=$ 0,012]; economia de R $\$ 694,20 / 100$ pacientes).

As despesas médias com exames e medicamentos por grupo de 100 pacientes, segundo os níveis de atividade física, são apresentadas na figura 2. No que se refere aos exames solicitados (ANOVA com $p=0,414$ ) e os gastos com medicamentos (ANOVA com $\mathrm{p}=0,272$ ), não houve diferenças significativas entre os grupos: Ativo, M. ativo e Sedentário.

Os custos com medicamentos foram divididos em dois tipos: (i) aqueles destinados ao tratamento do DM e (ii) o custo com medicamentos destinados ao tratamento de outras doenças (Figura 3). Quando comparado ao grupo Sedentário (ANOVA com $\mathrm{p}=0,001$ ), os resultados indicaram que os custos com medicamentos para o tratamento de outras doenças foram inferiores para os diabéticos do grupo Ativo (teste post hoc de Tukey $\mathrm{p}=0,006)$.

A figura 4 informa os custos totais dos pacientes diabéticos segundo o nível de atividade física e aponta que não houve diferenças significativas entre os grupos: Ativo, M. ativo e Sedentário (ANOVA com $\mathrm{p}=0,554$ ). Se considerado o custo total, na comparação entre diabéticos sedentários e ativos, a economia em um grupo de 100 pessoas seria de aproximadamente R $\$ 7.000,00$. 


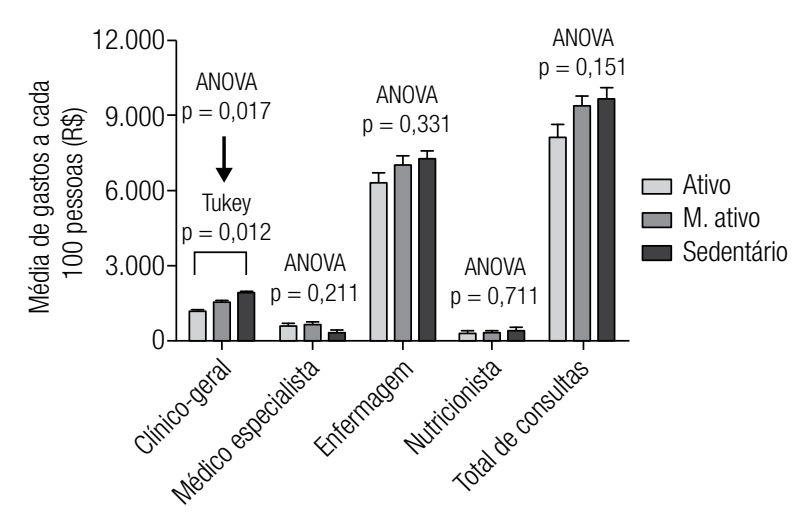

Procedimentos de atenção básica à saúde

M. ativo: moderadamente ativo; Total de consultas: Soma de clínico geral, médico especialista, enfermagem e nutricionista. Utilizou-se ANOVA one way.

Figura 1. Média \pm erro-padrão dos valores monetários de procedimentos de atenção básica a cada 100 pacientes, segundo níveis de atividade física, Bauru, 2009.

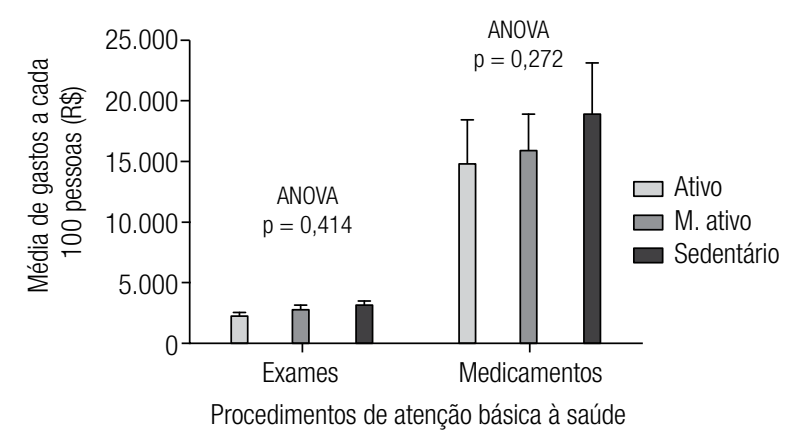

M. ativo: moderadamente ativo. Utilizou-se ANOVA one way.

Figura 2. Média \pm erro-padrão dos valores monetários de exames e medicamentos a cada 100 pacientes, segundo níveis de atividade física, Bauru, 2009.

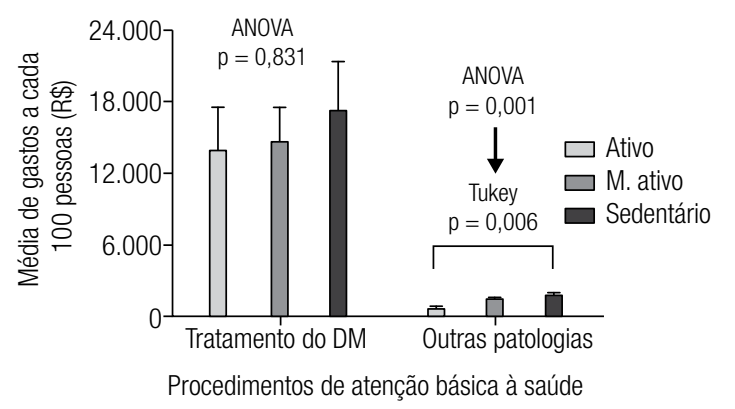

M. ativo: moderadamente ativo. Utilizou-se ANOVA one way.

Figura 3. Valores monetários médios \pm erro-padrão para custos com medicamentos para 0 diabetes e 0 tratamento de outras doenças, por 100 pacientes segundo nível de atividade física, Bauru, 2009.

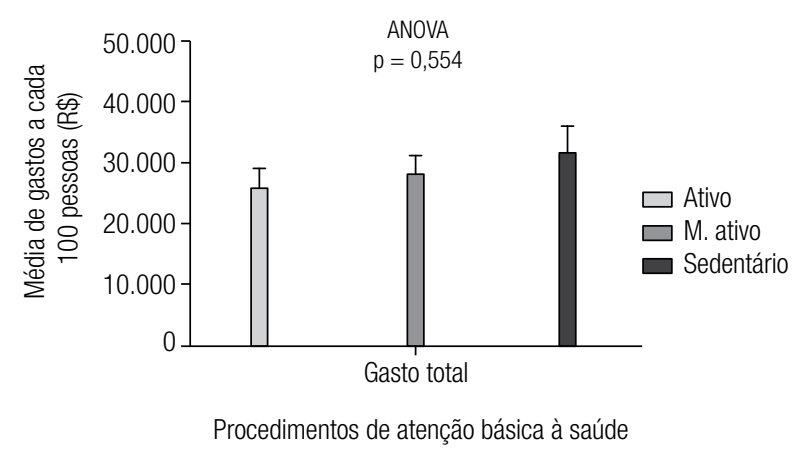

M. ativo: moderadamente ativo. Utilizou-se ANOVA one way.

Figura 4. Média \pm erro-padrão dos valores monetários de custos totais com procedimentos de atenção básica a cada 100 pacientes, segundo níveis de atividade, Bauru, 2009.

\section{DISCUSSÃO}

No que se refere à diminuição dos gastos com o tratamento decorrente da prática de atividades físicas, observamos que, mesmo em países em desenvolvimento, poucos estudos abordam esse tema. A esse propósito, Bertoldi e cols. (8), investigando a população de Pelotas-RS, observaram que indivíduos sedentários usaram mais medicamentos (39\%) que os ativos. Essas diferenças permaneceram mesmo quando incluídos os pacientes diabéticos e hipertensos. Na mesma direção, Di Loreto e cols. (11) analisaram o impacto do aumento do gasto energético de portadores de diabetes tipo 2 sobre indicadores de custo com a saúde. Após dois anos de intervenção, esses autores constataram que o custo por pessoa com medicamentos diminuiu significativamente, sendo que o maior gasto energético foi inversamente proporcional aos custos com tratamento farmacológico.

Os dados da presente pesquisa concordam com esses resultados. Os pacientes ativos apresentaram menores gastos com o tratamento farmacológico. Quando analisados separadamente (I) os fármacos utilizados para o tratamento do diabetes e (II) os fármacos utilizados para o tratamento de outras doenças, observou-se que o grupo Sedentário apresentou custo médio 23\% superior ao grupo Ativo, quando considerados apenas os medicamentos para o tratamento do diabetes (sem diferença estatística). Por outro lado, no que se refere ao uso de medicamentos para o tratamento de outras doenças, o grupo Sedentário apresentou custo $128 \%$ 
superior ao grupo Ativo, sendo essa diferença significativa estatisticamente.

Uma provável explicação para não ocorrer diminuição significativa nos custos com medicamentos para o tratamento do diabetes pode ser o fato de que muitos dos pacientes avaliados usam insulina, sendo o percentual de insulino-dependentes similar nos grupos de atividade física. Em decorrência da disfunção das células-beta, que reduz a produção de insulina de forma irreversível (5), seu uso injetável é constante e, mesmo com maior prática de atividades físicas, a necessidade de insulina exógena é necessária, sendo difícil a redução do medicamento e, consequentemente, do valor destinado ao tratamento. Nesse caso, estratégias de prevenção primária e secundária visando evitar que diabéticos tipo 2 se tornem insulino-dependentes caracterizam-se como políticas públicas que podem acarretar economia aos serviços de saúde e maior sobrevida para esses diabéticos.

No presente estudo, os pacientes ativos foram os que apresentaram despesas inferiores para clínico-geral quando comparados com os sedentários, que, por sua vez, tiveram custos $63 \%$ superiores, ou seja, R\$ 694,20 a mais, a cada 100 pacientes. Alguns efeitos do exercício físico são bem estabelecidos na literatura, como aumento da captação de glicose periférica e aumento da sensibilidade à insulina. É fato, também, que a atividade física influencia de maneira positiva nas funções cognitivas, transtornos de humor e sono, trazendo sensação de bem-estar (12). Essas variáveis, associadamente, podem ser responsáveis pela diminuição nas consultas médicas, uma vez que os pacientes têm uma autopercepção de melhora com o exercício físico e passam a procurar por serviços médicos apenas quando é realmente necessário.

Quanto aos usuários do serviço de saúde, as avaliações ocorreram após triagem realizada pelas enfermeiras, que analisavam o prontuário do paciente e sua disponibilidade em participar da pesquisa. Dessa forma, a casuística foi composta por casos, e os dados não representam a população de outras UBS de Bauru, sendo essa uma limitação do estudo. Outra limitação diz respeito ao questionário utilizado. O fato de o questionário de Baecke não possuir ainda um ponto de corte bem definido para classificação dos indivíduos em ativos e sedentários deve ser considerado como limitação do estudo, pois há poucos trabalhos publicados que também utilizaram a divisão em quartil para classificação do instrumento. Chien e cols. (13), por exemplo, utilizaram o mesmo questionário de atividade física e estratifica- ram sua amostra de acordo com o escore para atividades esportivas (quartil) comparando os escores observados em cada quartil. No quartil mais elevado, os valores encontrados foram similares aos observados no respectivo quartil da nossa casuística $(8,8$ vs. 8,9 , respectivamente). No entanto, ao se comparar o escore total médio de atividade física alcançado por nossa amostra (escore $=6,5$ ), constatou-se que este foi inferior ao apresentado no estudo de desenvolvimento do questionário (masculino $[\mathrm{n}=139]=8,2$ e feminino $[\mathrm{n}=167]=8,4) \mathrm{com}$ adultos holandeses saudáveis (10). Nossos resultados também foram inferiores aos observados em 29 adultos brasileiros residentes na cidade de São Paulo, no caso, sujeitos acometidos por HIV/Aids (escore = 7,3) $(14)$.

Entretanto, essa comparação é restrita uma vez que a população encontrada na literatura difere da casuística desse trabalho, que tem como fator de destaque a presença de DM2, dificultando, assim, identificar se os pacientes com maiores escores de atividade física são efetivamente suficientemente ativos. Por outro lado, o uso do questionário permitiu avaliar o nível de atividade física habitual total e em seus diferentes domínios, bem como apresentou boa aplicabilidade e aceitação.

A construção de um modelo teórico para projetar a abrangência dos resultados aqui amealhados demonstra que, se considerarmos o fato de que $6,2 \%$ da população do estado de São Paulo é acometida por DM2 (15), e que, destes, $47 \%$ são insuficientemente ativos (16), é possível estimar que há 21.551 pessoas acometidas pela doença na cidade de Bauru (347.601 habitantes), das quais 10.129 seriam insuficientemente ativas. Desse modo, considerando os parâmetros populacionais de Bauru, aplicados os dados da presente pesquisa, quando comparados ativos e sedentários, observa-se economia a cada 100 pessoas, de aproximadamente R\$ 6.500,00 reais/ano para os fisicamente ativos, assim, projeta-se uma economia de R\$ 658.385,00 reais/ano em custos com saúde se os 10.129 diabéticos sedentários se tornassem fisicamente ativos.

De fato, tal modelo constitui um raciocínio rústico dos possíveis benefícios financeiros decorrentes de uma maior prática de atividades físicas por parte de diabéticos do tipo 2 atendidos pelo SUS, porém, pode servir como ponto de partida para a implementação de diferentes ações municipais/estaduais nesse sentido. Além disso, essas reduções podem estar subestimadas em decorrência de a tabela de valores do SUS (base para o cálculo dos custos) ser inferior aos valores reais de mercado. 
Os dados apresentados permitem concluir que, ao analisar um período de 12 meses anterior ao momento de coleta das informações, pacientes diabéticos com escores superiores de atividade física diária apresentaram menores custos com serviços médicos e consumo de medicamentos. Além disso, mesmo nos casos em que as comparações estatísticas não expressaram diferenças estatisticamente significantes, quase todas as despesas tiveram custos mais elevados no grupo sedentário.

Declaração: os autores declaram não haver conflitos de interesse científico neste estudo.

\section{REFERÊNCIAS}

1. Federação Internacional de Diabetes. Atlas do diabetes, 2010. Disponível em: http://www.diabetesatlas.org/content/diabetes. Acesso em: 5 Ago 2010.

2. Sichieri R, do Nascimento $S$, Coutinho W. The burden of hospitalization due to overweight and obesity in Brazil. Cad Saude Publica. 2007;23(11):1721-7.

3. Cowie CC, Rust KF, Byrd-Holt DD, Eberhardt MS, Flegal KM, Engelgau MM, et al. Prevalence of diabetes and impaired fasting glucose in adults in the U.S. population: National Health and Nutrition Examination Survey 1999-2002. Diabetes Care. 2006;29(11):1263-8.

4. Assunção MC, Santos Ida S, Costa JS. Process assessment of health care: adequacy of the diabetes mellitus treatment in Pelotas, Southern Brazil. Cad Saude Publica. 2002;18(1):205-11.

5. Sociedade Brasileira de Diabetes. Tratamento e acompanhamento do diabetes mellitus: Diretrizes da Sociedade Brasileira de Diabetes, 2007. Disponível em: http://ww2.prefeitura.sp.gov.br//arquivos/secretarias/saude/programas/0007/Diretrizes_SBD_2007. pdf Acesso em: 13 Mar 2007.
6. Guedes DP, Gonçalves LA. Impact of the habitual physical activity on lipid profile in adults. Arq Bras Endocrinol Metabol. 2007;51(1):72-8.

7. Lima-Silva AE, Adami F, Nakamura FY, de Oliveira FR, Gevaerd MS. Metabolismo de gordura durante o exercício físico: mecanismos de regulação. Rev Bras Cineantrop Desempenho Hum. 2006;8(4):106-14.

8. Bertoldi AD, Hallal PC, Barros AJ. Physical activity and medicine use: evidence from a population-based study. BMC Public Health. 2006;6:224.

9. Rolim RM, Amaral SL, Monteiro HL. Hipertensão e exercício: custos do tratamento ambulatorial, antes e após a adoção da prática regular e orientada de condicionamento físico. Hipertensão. 2007;10(2):54-61.

10. Baecke JA, Burema J, Frijters JE. A short questionnaire for the measurement of habitual physical activity in epidemiological studies. Am J Clin Nutr. 1982;36(5):936-42.

11. Di Loreto C, Fanelli C, Lucidi P, Murdolo G, De Cicco A, Parlanti N, et al. Make your diabetic patients walk: long-term impact of different amounts of physical activity on type 2 diabetes. Diabetes Care. 2005;28(6):1295-302.

12. Mello MT, Boscolo RA, Esteves AM, Tufik S. O exercício físico e os aspectos psicobiológicos. Rev Bras Med Esporte. 2005;11(3):203-7.

13. Chien KL, Chen MF, Hsu HC, SuTC, LeeYT. Sports activity and risk of type 2 diabetes in Chinese. Diabetes Res Clin Pract. 2009;84(2):311-8.

14. Florindo AA, Latorre Mdo R, Santos EC, Negrão CE, Azevedo LF, Segurado AA. Validity and reliability of the Baecke questionnaire for the evaluation of habitual physical activity among people living with HIV/AIDS. Cad Saude Publica. 2006;22(3):535-41.

15. Schmidt MI, Duncan BB, Hoffmann JF, Moura L, Malta DC, Carvalho RM. Prevalence of diabetes and hypertension based on self-reported morbidity survey, Brazil, 2006. Rev Saude Publica. 2009;43(Suppl 2):74-82.

16. Silva TR, Feldman C, Lima MH, Nobre MR, Domingues RZ. Controle de diabetes mellitus e hipertensão arterial com grupos de intervenção educacional e terapêutica em seguimento ambulatorial de uma unidade básica de saúde. Saúde e Sociedade. 2006;15(3):180-9. 